volume was recorded before, during and after a 5 min occlusion period. The 'RH-PAT index' was then calculated by dividing the average digital pulse value measured during reactive hyperemia by that measured at baseline. The lower the RH-PAT index, the greater the extent of peripheral endothelial dysfunction.

A total of 94 patients were referred for coronary angiography to exclude significant coronary artery disease. All patients then underwent $\mathrm{RH}$-PAT and standard assessment of coronary vasoreactivity. Mean RH-PAT index was significantly lower in patients with coronary endothelial dysfunction than in those without $(P<0.001)$. Below a cut-off value of 1.35, the RH-PAT index had a sensitivity of $80 \%$ and a specificity of $85 \%$ to diagnose coronary endothelial dysfunction. The authors suggest that this approach might be a useful test to identify patients in the early stages of coronary artery disease.

Original article Bonetti PO et al. (2004) Noninvasive identification of patients with early coronary atherosclerosis by assessment of digital reactive hyperemia. J Am Coll Cardiol 44: $2137-2141$

\section{Advances in coronary angiography}

Although conventional coronary angiography remains the accepted technique for assessing coronary stenoses, advances in multi-slice computed tomography (MSCT) might offer a noninvasive alternative.

Mollet and colleagues from the Netherlands have tested the latest 16-slice MSCT scanner (Sensation16 Straton, Siemens, Forchheim, Germany) in 51 patients with stable angina or atypical chest pain. After intravenous injection of an iodinated contrast agent, MSCT coronary angiograms were taken during a mean scan time of $18.9 \pm 1.0 \mathrm{~s}$. The scans were interpreted by two observers masked to the findings on conventional coronary angiography and the results for the two methods were compared.

All patients with normal coronary arteries or significant $(\geq 50 \%)$ stenoses were correctly identified using MSCT coronary angiography, although this method incorrectly diagnosed single-vessel disease in 3 of 11 patients who had nonsignificant lesions. An analysis of the results from all proximal and mid-coronary segments showed that MSCT coronary angiography detected significant coronary lesions with $95 \%$ sensitivity, 98\% specificity, a positive predictive value of $87 \%$ and a negative predictive value of $99 \%$.

The investigators observe that MSCT coronary angiography is unlikely to replace traditional angiography in the short term. Since the technique is reliable, safe and noninvasive, however, it might be useful for certain applications, such as the assessment of asymptomatic individuals at high risk of atherosclerosis. Larger studies will be required to confirm these findings.

Original article Mollet NR et al. (2005) Improved diagnostic accuracy with 16-row multi-slice computed tomography coronary angiography. J Am Coll Cardiol 45: 128-132

\section{Does statin therapy prevent myocardial injury following PCI?}

Percutaneous coronary intervention (PCl) carries a risk of periprocedural myocardial injury. In a recent study by Mulukutla and colleagues, this risk appeared to be reduced by pretreatment with statins.

The authors carried out a retrospective study of 425 patients who underwent successful coronary stenting. The patients were divided into two groups: the statin group $(n=275)$, who had been receiving simvastatin, atorvastatin, pravastatin or lovastatin for at least one week before the procedure; and the control group $(n=150)$, who were not on statin therapy.

Analysis of venous blood samples taken 6-16 h after $\mathrm{PCl}$ revealed smaller postprocedural increases in creatine kinase (CK) in patients receiving statins that in those from the control group. CK increases of more than three times the upper limit of normalindicating that periprocedural myocardial infarction had occurred-were significantly less frequent in the statin group than in the control group ( $1.8 \%$ vs $6.0 \%$ of patients, $P=0.02$ ). Similar differences were found between the two groups in the proportion of patients who had more than three times the normal level of the isoenzyme CK-MB. Taken together, these results indicate a $70 \%$ relative reduction in the risk of significant myocardial injury in patients who receive statins before $\mathrm{PCl}$.

This work adds to the body of evidence suggesting that statin therapy exerts beneficial 